members of a class of students towards the end of an hour's lecture. On the other hand, pressure on the femoral artery and its sympathetic nerves, which, anatomically, run from the second and third lumbar nerves, is likely to be produced upon the bony brim of the pelvis by straightening the limb after prolonged flexion. The artery and its nerves pass over the prominence caused by the junction of the ilium and pubis, to which eminence the posterior wall of its sheath is bound down by the upper extension of the ilio-pectineal septum. It is probably here that the pressure effects are produced. Such pressure, on standing upright, would most likely be developed in individuals who from early years had been accustomed to prolonged hours of sitting.

It is extremely significant that the vascular lesion in these cases of intermittent claudication, which has been described by Dr. F. Parkes Weber ${ }^{2}$ in these words, "both the artery and one of the veins accompanying it were occluded by organising thrombi," is similar to that described by $\mathrm{Mr}$. Wingate Todd in the radial artery and accompanying veins in a case of cervical rib. ${ }^{3}$ It would, therefore, seem to be not unlikely that neuritic sciatica and intermittent claudication may represent the brachial neuritis and vascular symptoms of cervical rib cases; that both are pressure effects produced by the passage of structures over the pelvic bones, but that opposed conditions produce the two sets of symptoms.

\section{AN UNUSUAL ABDOMINAL CASE.}

\section{BY JOHN AIKMAN, M.D., C.M. GLASG.}

A MARRIED woman, well advanced in her forty-third year, summoned me to relieve her of a retention of urine. I found her bladder hugely distended, and I emptied it by the catheter. I had not seen her for about two years, but I had previousiy told her of the existence of a uterine fibroid which had not seemed to grow much and which I hoped would never call for operation. She told me, at my visit, that she had suffered from retention of urine for some time, but that with waiting and trying she had always previously got relief. When the bladder was emptied I found the old fibroid, of about the size of a billiard ball, near the right cornu of the uterus, and about the same size as on former occasions. But the abdomen was filled up to nearly the level of the umbilicus with a firm but more soft mass which was new and not easy of explanation. 'The patient's temperature was slightly raised-99.4० $\mathrm{F}$. The pelvis was filled with the same mass which protruded into the abdomen, the main location being in Douglas's pouch. The os uteri was patulous and the posterior wall was bulged towards the uterine cavity. The whole organ was lower in the pelvis than is normal. The patient had a son, now in his seventeenth year; her periods were regular-in fact, the recent attack of retention had begun during her last period in the few days preceding my visit. In the evening retention again took place and was relieved by the catheter. Next day she was more easy and passed urine, but did not quite empty the bladder. She was told that no further information conld be given without an exploratory incision, and as she wished this done without delay it was performed on the fourth day after she was first seen.

After due preparation the abdomen was opened through the right rectus and the cavity was found unduly moist, but without any such collection of fluid as could be called ascites. The old fibroid was there as described, and the ovaries were manifestly degenerating. The mass which bulged up from Douglas's pouch was shut off from the general cavity of the peritoneum, but was attached to the uterus nearly as high as the site of the fibroid. It was thought unwise to open it into the general cavity, so the ovaries were tied off and removed, and the cavity closed for the time with catch forceps. The anterior lip of the os uteri was fixed with vulsellum forceps, and the posterior lip incised into the bulge, in the middle line, and back into the base of the swelling. Copious watery discharge cozed from the cut surfaces, and with great care masses of pale-coloured tissue

2 The LANCE T, Jan. 18th, 1913, p. 169.

3 The LANGet, August 10th, 1912, and Journal of Anatomy and 3 THE LANCET, August
Physiology, January, 1913. resembling an old placenta were extracted. Many portions were almost as hard as a soft fibroid, while the less hard portions showed none of the characters of a recent placenta. The higher portions of the mass came down more easily, apparently indicating that the unfound foetus had escaped from below; but the higher portion was so intimately attached to the uterus that the fundus came down into the vaginal wound and was removed. The utero-vaginal stump was brought together, but the opening into the sequestered placental sac was left open for drainage. The completeness of the isolation of the peritoneal cavity was verified from the abdominal wound, which was then closed. A catheter was then passed and urine free from blood-stain was drawn off. The operation was long and tedious, but the promise seemed to be good. The catheter was prescribed to prevent any soiling of the vaginal wound, but this was not accomplished. The vaginal discharge was very copious and soon became odorous. The vagina was douched with the hope of securing against any infection of the higher parts, and the abdominal wound healed without any sign of infection of the peritoneal cavity. But the patient sank and died during the twelfth day after operation.

No information can be obtained as to the date of this relic of an extra-uterine pregnancy, but the placenta was firmer and harder than was the placenta in one case in which a similar vestige was removed six years after the date of a pelvic hæmatocele. The patient was a hardworking woman, and the probable explanation is that the contraction of the placenta in its lower regions caused the retention of urine and the descent of the womb. It is not difficult to understand that the retained urine spread some infection to the placental remains, which were obviously on the verge of inflammatory change. I hope that the operative interference gave the patient the more safe prospect.

My acknowledgments are due to Mr. A. C. Wallace and Dr. M. Ross Taylor, who assisted me.

Guernsey.

A CASE OF GANGRENE OF THE LEG IN AN INFANT 11 DAYS OLD; AMPUTATION; RECOVERY.

\section{By DUNCAN O. L. FITZWILliams, Ch.M. Edin.,} F.R.C.S. ENG. \& EDIN.,

SURGEOY TO OUT-PATIENTS, ST. MARX'S HOSPITAT, AND THE HOSPITAT FOR SICK CHITDREN, PADDIXGTON GREEN;

$$
\text { AND }
$$

W. H. VINCENT, L.M.S.S.A. LOND.,

LATK RESIDENT OBSTETRIC OFFICER, ST. MARY'S HOSPITAL.

THE occurrence of gangrene of a large portion of the limb of an infant under a month old must be such a rare occurrence as to justify the publication of the following case.

A boy was born on June 20th, 1912, the first child of apparently healthy parents. The labour was quite natural, the presentation was a normal vertex, and no assistance was required; indeed, the child was born before the arrival of a nurse or medical man. A neighbour, however, was present, and the medical practitioner arrived in time to tie the cord. The child was apparently quite well until June 30th, on which date a small blue patch appeared on the outer side of the calf of the right leg. The area of discolouration gradually became larger, and by the next day had spread down to the ankle. By July 2nd it had reached the outer toes. On the 5th the child, now 14 days old, was taken by his father to St. Mary's Hospital. The condition was then as described below.

The lower two-thirds of the right leg were dusky blue; immediately above this level the skin was red, and gradually faded into the natural colour just below the knee. The line between the two areas wandered in an irregular manner right round the limb. The right limb was much colder than the left, but was not devoid of heat, though probably this was communicated to it from the opposite leg. There seemed to be no circulation in the discoloured portion, although the big toe and the adjacent portion of the foot 